\title{
АНАЛИЗ СМЕСЕЙ АНИОНОВ МЕТОДОМ ОДНОКОЛОНОЧНОЙ ИОННОЙ ХРОМАТОГРАФИИ: ИСПЫТАНИЕ СОРБЕНТА ТИПА ВАКС
}

\author{
(Представил О. Эйзен)
}

Ионная хроматография - широко используемый аналитический метод определения многих анионов и катионов. В ионной хроматографии разделение определяемых ионов осуществляется на специальных ионитах (сорбентах) с низкой обменной емкостью [1, 2]. Разработано два варианта этого метода: двухколоночный [ $\left.{ }^{1}\right]$ и одноколоночный $\left.{ }^{2}\right]$. В первом случае поток элюента пропускается после разделяющей колонки через т. н. компенсационную колонку $\left[{ }^{3}\right]$ в целях подавления электропроводности элюента. Низкая фоновая электропроводность элюента способствует достижению высокой чувствительности кондуктометрического детектора, генерирующего регистрируемый аналитический сигнал. В одноколоночном варианте ионной хроматографии, где компенсационная колонка отсутствует $\left[{ }^{2}\right]$, используются элюенты с низкой электропроводностью, либо кондуктометрический детектор в нем заменяется на ультрафиолетовый абсорбционный $\left[{ }^{4}\right]$. Для рутинной аналитической работы более подходящим можно считать одноколоночный вариант ионной хроматографии, так как в этом случае отпадает необходимость в периодической регенерации компенсационной колонки. Следует, однако, отметить, что уже появились и устройства непрерывного действия для подавления электропроводности элюентов [5,6.

Метод ионной хроматографии особенно эффективен для определения анионов. Недавно предложены новые оригинальные отечественные сорбенты для разделения смесей анионов [7,8]. В целях опробования одного из них - сорбента типа ВАKC $\left.{ }^{8}\right]$ - в настоящей работе изучены зависимости эффективности и селективности колонок, заполненных указанным сорбентом, от трех факторов: скорости подачи элюента, концентрации фталатного элюента и обменной емкости сорбента.

\section{Экспериментальная часть}

Исследования проводили на ионном хроматографе IVK-11 с кондуктометрическим детектированием ионов, сконструированном в СКБ АН ЭССР. Постоянная ячейка детектора $3,0 \mathrm{~cm}^{-1}$. Фоновые проводимости элюентов были в пределах 26-48 мкСм, аналитический сигнал регистрировали в диапазоне $0-100$ мкСм (соответствует выходному сигналу 10 мВ, подаваемому на самопишущий электронный потенциометр).

Колонки из нержавеющей стали длиной 150 мм и внутренним диаметром 2 мм заполняли под давлением 8-9 МПа сорбентами, суспендированными во фталатном элюенте.

Сорбенты типа ВАКС синтезировали по методике [87. Исходным материалом служил сферон 100000 фирмы «Lachema» (ЧCCP), фракщия 
25-40 мкм. Соотношение клеящее вещество:анионит поддерживали

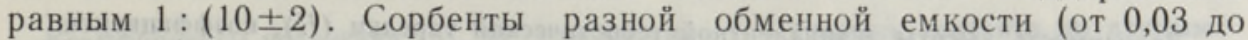
0,15 мэкв/г) получали путем добавления соответствующих количеств растворов клеящего вещества (коллоксилина ПСВ) и анионита (BA-2) к сферону 100000 .

В качестве элюентов использовали водные растворы фталата калия ч. д. а. с концентрацией от 0,5 до 1,5 м М. Хроматографические колонки испытывали стандартным раствором, содержащим ионы хлорида $\left(5 \mathrm{~m} /\right.$ дм $\left.^{3}\right)$, нитрата $\left(10 \mathrm{мг} /\right.$ дм $\left.^{3}\right)$ и сульфата $\left(15 \mathrm{мг} /\right.$ дм $\left.^{3}\right)$. Объем вводимой пробы 100 мм³ $^{3}$. Стандартный анализируемый раствор приготовляли из соответствующих натриевых солей ч.д. а.

Обменную емкость колонок определяли по двум методикам. По методике А смолу переводили в карбонатную форму и промывали деионизированной водой для удаления остатка карбоната. Емкость смолы определяли, переводя навеску сорбента из карбонатной формы в хлоридную промыванием $1-2$ м $\mathrm{HCl}$. При этом учитывали объем колонки, занимаемый элюентом. По методике Б смолу переводили в нитратную форму, промывали деионизированной водой и замещали нитрат ионами фталата. Элюированное количество нитрата определяли методом ионной хроматографии.

\section{Результаты и их обсуждение}

Для выявления влияния объемной скорости элюента изучали зависимость высоты эквивалентной теоретической тарелки (ВЭТТ) от скорости подачи элюента [9] (табл. 1). Для оценки разделяющей способности колонки выгисляли три величины: относительное удерживание

$$
\alpha_{\mathrm{NO}_{3}^{-} / \mathrm{SO}_{4}^{2-}}=\left(t_{R 2}-t_{0}\right) /\left(t_{R 1}-t_{0}\right),
$$

где $t_{R 2}$ и $t_{R 1}$ - времена удерживания соответственно сульфат- и нитратионов, мин, $t_{0}$ - время удерживания несорбирующего иона, мин; массовое отношение распределения (коэффициент емкости $k^{\prime}$ ) $\left[{ }^{9,10}\right]$

$$
k^{\prime}=\left(t_{R}-t_{0}\right) / t_{0}
$$

и разделение

$$
R_{\mathrm{NO}_{3}^{-} / \mathrm{SO}^{2-}}=2\left(t_{R 2}-t_{R 1}\right) /\left(\omega_{2}-\omega_{1}\right),
$$

где $\omega_{2}$ и $\omega_{1}$ - ширины пиков соответствующих ионов у основания.

Исследования показали, что эффективность колонки снижается почти вдвое при увеличении объемной скорости от 0,3 до $1,5 \mathrm{~cm}^{3} / \mathrm{Mnн}$. При этом время анализа уменьшается с 9,5 до 2,1 мин. Вычисленное по уравнению (3) разделение [ $\left.{ }^{9}\right]$ возрастает от 1,75 при $1,5 \mathrm{~cm}^{3} /$ мин до 2,36 при $0,3 \mathrm{~cm}^{3} /$ мин. Разделение улучшается за счет повышения эффективности колонки. Следует отметить, что селективность колонки остается постоянной в пределах ошибок эксперимента (см. табл. 1).

Для изучения второй зависимости проводили серию опытов при постоянной скорости элюента, но с изменением его концентрации от 0,5 до 1,5 мМ. Согласно уравнению

$$
\lg \left(t^{\prime}\right)=(-y / x) \lg c+y / x \lg (\text { емк. })-C,
$$

где $y, x-$ заряды элюированного и элюирующего анионов соответственно, $C$ - константа, логарифм исправленного времени удерживания $\left(t^{\prime}\right.$, мин) линейно связан с логарифмом концентрации элюента $(c)$ при постоянстве обменной емкости смолы (емк., мэкв/г), объемной скорости и $\mathrm{pH}\left[{ }^{10}\right]$. 
Зависимость высоты эквивалентной теоретической тарелки $(H)$, коэффициента емкости $\left(\kappa^{\prime}\right)$, относительного удерживания $(\alpha)$ и разделения $(R)$

от объемной скорости $(F)$ элюента

У сло в и я: колонка $150 \times 2$ мм, емкость смолы ВАКС 0,10 мэкв/г, элюент - фталат калия 1 мМ, рН 5,2 (здесь и в табл. 2 и 5).

\begin{tabular}{|c|c|c|c|c|c|c|c|}
\hline \multirow{2}{*}{$\begin{array}{c}F, \\
\mathrm{~cm}^{3} / \text { мин }\end{array}$} & \multicolumn{2}{|c|}{$\mathrm{NO}_{3}^{-}$} & \multicolumn{2}{|c|}{$\mathrm{SO}_{4}^{2-}$} & \multirow{2}{*}{$\mathrm{RNO}_{3}^{-} / \mathrm{SO}_{4}^{2-}$} & \multirow{2}{*}{$\alpha_{\mathrm{NO}_{3}^{-}}^{-} / \mathrm{SO}_{4}^{2-}$} & \multirow{2}{*}{\begin{tabular}{|c} 
Полное \\
время \\
анализа, \\
мин
\end{tabular}} \\
\hline & $H$, мм & $\kappa^{\prime}$ & $H$, м м & $\kappa^{\prime}$ & & & \\
\hline $\begin{array}{r} \\
1,5 \\
1,3 \\
1,1 \\
0,9 \\
0,7 \\
0,5 \\
0,3\end{array}$ & $\begin{array}{l}0,58 \\
0,54 \\
0,48 \\
0,41 \\
0,38 \\
0,36 \\
0,31\end{array}$ & $\begin{array}{l}2,8 \\
2,7 \\
2,8 \\
2,8 \\
2,9 \\
2,9 \\
3,0\end{array}$ & $\begin{array}{l}0,67 \\
0,64 \\
0,54 \\
0,51 \\
0,47 \\
0,41 \\
0,38\end{array}$ & $\begin{array}{l}5,7 \\
5,7 \\
5,6 \\
5,7 \\
6,0 \\
5,7 \\
5,7\end{array}$ & $\begin{array}{l}1,75 \\
1,87 \\
1,98 \\
2,03 \\
2,07 \\
2,35 \\
2,36\end{array}$ & $\begin{array}{l}2,0 \\
2,1 \\
2,0 \\
2,0 \\
2,0 \\
2,0 \\
1,9\end{array}$ & $\begin{array}{l}2,1 \\
2,4 \\
2,8 \\
3,3 \\
4,1 \\
5,8 \\
9,5\end{array}$ \\
\hline
\end{tabular}

Таблица 2

Исправленные времена удерживания $\left(t^{\prime}\right)$, высота эквивалентной теоретической тарелки $(H)$, коэффициенты емкости $\left(\kappa^{\prime}\right)$ и относительное удерживание $(\alpha)$ на смоле ВАКС (емкость 0,10 мэкв/г) при объемной скорости $0,9 \mathrm{~cm}^{3} /$ мин

\begin{tabular}{|c|c|c|c|c|c|c|c|c|}
\hline \multirow{2}{*}{$c, \mathrm{MM}$} & \multicolumn{3}{|c|}{$\mathrm{NO}_{3}^{-}$} & \multicolumn{3}{|c|}{$\mathrm{SO}_{4}^{2-}$} & \multirow{2}{*}{$\alpha_{\mathrm{NO}_{3}^{-}} / \mathrm{SO}_{4}^{2-}$} & \multirow{2}{*}{$\begin{array}{c}\text { Полное } \\
\text { время } \\
\text { анализа } \\
\text { мин }\end{array}$} \\
\hline & $t^{\prime}$, мин & $H$, м м & $\kappa^{\prime}$ & $t^{\prime}$, мин & $H$, мм & $\kappa^{\prime}$ & & \\
\hline $\begin{array}{l}0,5 \\
1,0 \\
1,5\end{array}$ & $\begin{array}{l}4,82 \\
3,55 \\
2,88\end{array}$ & $\begin{array}{l}0,33 \\
0,31 \\
0,28\end{array}$ & $\begin{array}{l}3,86 \\
2,84 \\
2,30\end{array}$ & $\begin{array}{c}11,8 \\
7,15 \\
4,80\end{array}$ & $\begin{array}{l}0,39 \\
0,38 \\
0,39\end{array}$ & $\begin{array}{l}9,44 \\
5,78 \\
3,84\end{array}$ & $\begin{array}{l}2,45 \\
2,04 \\
1,67\end{array}$ & $\begin{array}{c}15 \\
9,5 \\
6,8\end{array}$ \\
\hline
\end{tabular}

Результаты, полученные в этой серии экспериментов (табл. 2), говорят о том, что ВЭТТ не зависит от концентрации элюента в пределах ошибок измерения $\left(s_{R}=0,02\right)$. Относительное удерживание $\alpha_{\mathrm{NO}_{3}^{-} / \mathrm{SO}_{4}^{2-}}$ растет по мере убывания концентрации элюента, что связано с различными углами наклона зависимостей $\lg \left(t^{\prime}\right)=f(c)$ для нитрат- и сульфатионов.

Преобразовав уравнение (4), для расчета регрессионной кривой $t^{\prime}=f(c)$ получим

$$
y_{i}=A+B x_{i},
$$

где $A=x / y \lg$ (емк.) $-C, B=-y / x$ (табл. 3 ).

Если считать, что при $\mathrm{pH} 5,2$ элюирующий анион находится в форме двухзарядного фталата, то теоретическое значение коэффициента $B$ равно 1 для двухзарядного иона (сульфата) и 0,5 для однозарядного иона (нитрата) [ $\left.{ }^{10}\right]$. Как видно из табл. 3 , вычисленные значения $B$ действительно близки к указанным.

Третью зависимость изучали на колонках одинаковых размеров $(150 \times 2$ мм), заполненных смолами разной обменной емкости. При этом емкость сорбента измеряли по методикам А и Б (табл. 4). Различие в результатах оказалось статистически незначимым. 
Коэффициенты уравнения (5), их стандартные отклонения $\left(s_{B}\right.$ и $\left.s_{A}\right)$, коэффициенты корреляции $(r)$ и отношение общей дисперсии $\left(s_{0}\right)$ к диапазону измерения $\left.y(\Delta y){ }^{11}\right]$

\begin{tabular}{c|c|c|c|c}
\hline Ион & $B \pm s_{B}{ }^{*}$ & $A \pm s_{A}$ & $r$ & $\mathrm{~s}_{0} / \Delta \mathrm{y}$ \\
\hline $\mathrm{NO}_{3}^{-}$ & $0,494 \pm 0,084$ & $0,056 \pm 0,017$ & 0,986 & 0,128 \\
$\mathrm{SO}_{4}^{2-}$ & $0,927 \pm 0,100$ & $0,357 \pm 0,021$ & 0,994 & 0,087
\end{tabular}

* Число степеней свободы $f=2$.
Емкость сорбента по данным двух методик, мэкв/г

\begin{tabular}{|c|c|c|}
\hline \multirow{2}{*}{$\begin{array}{l}\text { Номер } \\
\text { смолы } \\
\text { ВАКС }\end{array}$} & A & Б \\
\hline & eмk. $\pm s_{\mathrm{A}} *$ & емк. $\pm s_{\text {Б }}$ \\
\hline 1 & $0,095 \pm 0,013$ & $0,100 \pm 0,008$ \\
\hline 2 & $0,051 \pm 0,014$ & $0,071 \pm 0,002$ \\
\hline 3 & $0,030 \pm 0,005$ & $0,035 \pm 0,004$ \\
\hline 4 & $0,148 \pm 0,013$ & $0,151 \pm 0,005$ \\
\hline
\end{tabular}

* $s$ А. Б- стандартное отклонение, четыре параллельных определения.

Таблица 5

Влияние емкости сорбента ВАКС на время удерживания $\left(t^{\prime}\right)$, высоту эквивалентной теоретической тарелки $(H)$ и коэффициент емкости $\left(\kappa^{\prime}\right)$

\begin{tabular}{|c|c|c|c|c|c|c|c|c|c|c|}
\hline \multirow{2}{*}{$\begin{array}{c}\text { Емкость, } \\
\text { мэкв } / \text { г }\end{array}$} & \multicolumn{3}{|c|}{$\mathrm{Cl}^{-}$} & \multicolumn{3}{|c|}{$\mathrm{NO}_{3}^{-}$} & \multicolumn{3}{|c|}{$\mathrm{SO}_{4}^{2-}$} & \multirow{2}{*}{$\begin{array}{c}\text { Полное } \\
\text { время ана- } \\
\text { лиза, мин }\end{array}$} \\
\hline & $\mid t^{\prime}$, мин $\mid$ & $H, \mathrm{~mm}$ & $\kappa^{\prime}$ & $\mid t^{\prime}$, мин & $H$, м м & $\kappa^{\prime}$ & $\mid t^{\prime}$, мин $\mid$ & $H, \mathrm{~m} \mathrm{м}$ & $\kappa^{\prime}$ & \\
\hline $\begin{array}{l}0,151 \\
0,100 \\
0,071 \\
0,035\end{array}$ & $\begin{array}{l}1,23 \\
0,84 \\
0,63\end{array}$ & $\begin{array}{l}0,54 \\
0,28 \\
0,31\end{array}$ & $\begin{array}{l}1,97 \\
1,34 \\
1,01\end{array}$ & $\begin{array}{l}3,01 \\
1,83 \\
1,18 \\
0,75\end{array}$ & $\begin{array}{l}0,52 \\
0,38 \\
0,48 \\
0,50\end{array}$ & $\begin{array}{l}4,80 \\
2,93 \\
1,89 \\
1,20\end{array}$ & $\begin{array}{l}6,93 \\
3,68 \\
2,63 \\
1,55\end{array}$ & $\begin{array}{l}0,48 \\
0,47 \\
0,58 \\
0,71\end{array}$ & $\begin{array}{l}11,1 \\
5,89 \\
4,21 \\
2,48\end{array}$ & $\begin{array}{l}8,55 \\
4,46 \\
3,45 \\
2,50\end{array}$ \\
\hline
\end{tabular}

$\overline{П р и м е ч ~ а ~ н ~ и е . ~ Д л я ~ х л о р и д и о н а ~ п р и ~ е м к о с т и ~} 0,035$ мэкв/г измерить эти величины не удается ввиду совмещения системного и определяемого пиков.

Повышенные значения коэффициента емкости получены с анионитами, обладающими более высокой емкостью. По данным табл. 5 можно определить необходимую емкость сорбента в зависимости от требуемого значения коэффициента емкости, которым определяется быстрота разделения компонентов. В большинстве случаев оптимальным считается $1 \leqslant \kappa^{\prime} \leqslant 4\left[{ }^{9}, 10\right]$.

\section{Выводы}

1. Синтезировано четыре сорбента типа ВАКС с обменной емкостью 0,035-0,151 мэкв/г. Изучены эффективность и селективность хроматографических колонок, заполненных этими сорбентами.

2. В хроматографических колонках с внутренним диаметром 2 мм уменьшение объемной скорости фталатного элюента от 1,5 до $0,3 \mathrm{~cm}^{3} /$ мин приводит к монотонному убыванию высоты эквивалентной теоретической тарелки (от 0,63 до 0,35 мм). При этом полное время анализа возрастает в 4,5 раза.

3. Эффективность хроматографических колонок не зависит от концентрации фталатного элюента в пределах от 0,5 до 1,5 мМ. Снижение концентрации элюента в указанных пределах приводит к росту селективности колонки в 1,5 раза. Она вычисляется по значениям относительного удерживания. 
1. Small. H., Stevens, T. S., Bauman, W. S. Novel ion exchange chromatographic method using conductimetric detection. - Anal. Chem., 1975, 47, 1801-1809.

2. Gjerde, D. T., Fritz, J. S., Schmuckler, G. Anion chromatography with low-conductivity eluents. - J. Chromatogr., 1979, 186, 509-519.

3. Small, H. Modern inorganic chromatography. - Anal. Chem., 1983, 55, 235A-236A, $238 \mathrm{~A}, 240 \mathrm{~A}, 242 \mathrm{~A}$.

4. Haddad, $P$. R., Heckenberg, A. L. Determination of inorganic anions by high-performance liquid chromatography. - J. Chromatogr., 1984, 300, 357-394.

5. Stevens, S. T., Davis, J. C. Hollow fiber ion-exchange suppressor for ion chromatography. - Anal. Chem., 1981, 53, 1488-1492.

6. Dasgupta, $P, K$. Ion chromatographic separation of anions with ion interaction reagents and an annular helical suppressor. - Anal. Chem., 1984, 56, 769-772.

7. Долгоносов А. М. Ионная хроматография на центрально-привитом анионообменнике. - Ж. физ. хим., 1984, 58, № 8, 1989-1991.

8. Haldna, O., Palvadre, R., Pentchuk, J., Kleemeier, T. Preparation of low-capacity anion-exchange resins for ion chromatography on a methacrylic copolymer matrix. - J. Chromatogr., 1985, 350, 296-298.

9. Энгельгарт X. Жидкостная хроматография при высоких давлениях. М., 1980, $14-35$.

10. Фриц Дж., Гьерде Д., Поланд К. Ионная хроматографня. М., 1984, 126-128.

11. Чарыков А. К. Математическая обработка результатов химического анализа. Л., $1984,141-144$.

Тартуская городская санэпидстанция

Ннститут химии

Академии наук Эстонской ССР

Поступила в редакцию $19 /$ XII 1985

\section{J. PENTSUK, O. HALDNA, K. ILMOJA}

\section{ANIOONIDE SEGUDE ANALUUS OHEKOLONNILISE IOONKROMATOGRAAFIA MEETODIL: BAKC-TUUPI SORBENDI KATSETAMINE}

Valmistati BAKC-tüüpi madala mahtuvusega $(0,03-0,15 \mathrm{mekv} / \mathrm{g})$ anioniite ja uuriti nende kromatograafilisi omadusi. Sealjuures kasutati ühekolonnilist ioonkromatograafilist aparatuuri, kus kolonni mõôtmed olid $150 \times 2 \mathrm{~mm}$ ja ioonide detekteerimine toimus konduktomeetriliselt. Iseloomustati kolme muutuja môju lahutava kolonni efektiivsusele ja selektiivsusele. Need muutujad olid: eluendi voolu mahtkiirus $\left(0,3-1,5 \mathrm{~cm}^{3} / \mathrm{min}\right)$, eluendi kontsentratsioon $(0,5-1,5 \mathrm{mM}$ kaaliumftalaat $)$ ja anioniidi mahtuvus $(0,035-0,151$ mekv/g) . Leiti, et kolonni efektiivsus kasvas kaks korda, kui eluendi mahtkiirust vähendati viis korda $\left(1,5 \mathrm{~cm}^{3} / \mathrm{min}\right.$ kuni $\left.0,3 \mathrm{~cm}^{3} / \mathrm{min}\right)$. Eluendi kontsentratsiooni muutumine piirkonnas $0,5-1,5 \mathrm{mM}$ ei avaldanud märgatavat mōju kolonni efektiivsusele. Kuid kolonni selektiivsus kasvas $\mathrm{ca}$ poolteist korda, kui eluendi kontsentratsioon vähenes mainitud piirides. Anioniidi mahtuvuse muutmine uuritud piirkonnas ei môjutanud oluliselt kolonni efektiivsust, kuid suurema mahtuvusega anioniitide $(0,151 \mathrm{mekv} / \mathrm{g})$ korral oli kolonni selektiivsus suurem, võrreldes väikese mahtuvusega anioniidiga $(0,035 \mathrm{mekv} / \mathrm{g})$.

\section{J. PENTCHUK, O. HALDNA, K. ILMOJA}

\section{DETERMINATION OF ANIONS BY SINGLE-COLUMN ION CHROMATOGRAPHY. ESTIMATION OF BAKC-TYPE RESINS PERFORMANCE}

BAKC-type anion exchanger resins of a low capacity $(0.03-0.15 \mathrm{meq} / \mathrm{g})$ have been prepared. The chromatographic performance of these resins was estimated using a nonsuppressed ion chromatographic system with a $150 \times 2 \mathrm{~mm}$ column and conductometric detector. The influence of three variables on the efficiency and selectivity of the separator column has been studied. The parameters varied were: eluent flow-rate $\left(0.3-1.5 \mathrm{~cm}^{3} / \mathrm{min}\right)$; eluent concentration $(0.5-1.5 \mathrm{mM}$ potassium phthalate; and resin capacity $(0.035-$ $0.151 \mathrm{meq} / \mathrm{g}$ ). It has been found that column efficiency increases twice if eluent flow-rate drops from 1.5 to $0.3 \mathrm{~cm}^{3} / \mathrm{min}$. The concentration of the eluent used showed no remarkable influence on the column efficiency, but column selectivity increased with decreasing eluent concentration (about one and a half times in the range studied). The changes in resin capacity have not influenced column efficiency but higher selectivity values were obtained with higher resin capacity $(0.151 \mathrm{meq} / \mathrm{g})$. 